\title{
Crossed avoiding reaction: a disturbance of the manual spatial function
}

\author{
Toyoko Nagumo, Atsushi Yamadori, Yoshiaki Soma, Ryoji Kayamori, Masakazu Ito
}

\begin{abstract}
A patient with MRI confirmed lesions in the corpus callosum and the left cingulate gyrus had a rare syndrome of crossed avoiding reaction of the left hand. With the right hand she could reach a stimulus object in whatever space it was presented. With the left hand, however, she could not mobilise it to reach a stimulus presented in the right hemispace relative to her body axis. In the left hemispace relative to her body axis her left hand reached an object without any difficulty. This left unilateral difficulty in the right unilateral space may be related to a unique spatial function that controls manual space, which is represented differentially in the two hemispheres. In the left hemisphere this function covers bilateral hemispace and is operated by the right hand. In the right hemisphere this function covers only the left hemispace and is operated by the left hand.
\end{abstract}

$(\mp$ Neurol Neurosurg Psychiatry 1993;56:552-555)

Department of Speech Therapy, Niigata Prefectural Muikamachi Hospital, Niigata, Japan

T Nagumo

Neurology Service, Hyogo Brain and Heart Center at Himeji, Japan A Yamadori

Department of Neurology, Niigata University, School of Medicine, Niigata,

Japan

Y Soma

Department of Rehabilitation, Teikyo University School of Medicine, Tokyo,

Japan

R Kayamori

Department of

Internal Medicine,

Niigata Prefectural

Nuigata Prefectural

Niigata, Japan

$M$ Ito

Correspondence to: Ms Toyoko Nagumo, Department of Speech Therapy, Niigata Prefectura Muikamachi Hospital, Muikamachi 636-2, Minamiuonuma-Gun, Niigata, 949-66, Japan

Received 16 January 1992 and in revised form 1 June

Accepted 18 August 1992 scan of the brain at the time showed no abnormality.
On 1 March 1988, she developed right sided weakness and was admitted to hospital on 3 March. She showed slight impairment of consciousness, mild right hemiparesis, and increased right tendon reflexes. On 20 December 1988 she was alert and cooperative. A left hemianopsia was not confirmed with Goldmann perimetry. Cranial nerves 3-12 were normal. No apparent motor weakness was present. Muscle tone was normal. However, movements of the left upper and lower extremities were clumsy in the fingerto-nose and heel-to-shin tests. Biceps and patellar tendon reflexes were increased, but no pathological reflexes were detected. Sensation was normal except for joint position sensation at the left fingers.

\section{Neuroimaging studies}

A CT scan of the brain revealed circumscribed low absorption areas in the corpus callosum from the genu through the body and in the adjacent medial area of the left frontal lobe consistent with a diagnosis of infarction of the left anterior cerebral artery. An MRI study was also performed. T1 weighted sagittal and coronal sections showed atrophy of the left half of the corpus callosum from the genu through the entire length of the body (fig 1). Proton weighted axial images revealed a low signal area in the left cingulate gyrus (fig 2). T2 weighted axial sections showed additional small lesions in the bilateral thalamus and in the left frontal subcortical area.

\section{Neuropsychological studies}

She looked vague but retained all the abilities for activities for daily living. Language was considered essentially normal although some paraphasias were noted in naming picture cards. WAIS performed one month after admission revealed verbal IQ of 60 and performance IQ was off scale. This poor score was somewhat surprising in view of her overall demeanour. During the course of examination it became clear that she had an array of callosal disconnection syndromes. Thus her left hand showed diagnostic apraxia, alien hand sign with self-grasping, tactile anomia, agraphia, and ideomotor apraxia. On copying pictures with the right hand she demonstrated left unilateral spatial neglect. Also on reading and naming pictures she neglected leftward stimuli. In raising both hands, motor initiation difficulty of the left hand was observed. We also noted that she had some difficulty 
Figure 1 T1 weighted MRI image (TR 600; TE 20). Sagittal section $3 \cdot 5$ mm left of the midline. At this level atrophy of the corpus callosum from the genu to the posterior third of the body is clearly seen.

Figure 2 Axial MRI image with proton emphasis (TR 2500; TE 15). At this level medial aspect of the left hemisphere (on the left) shows low signal area corresponding to the cingulate gyrus.
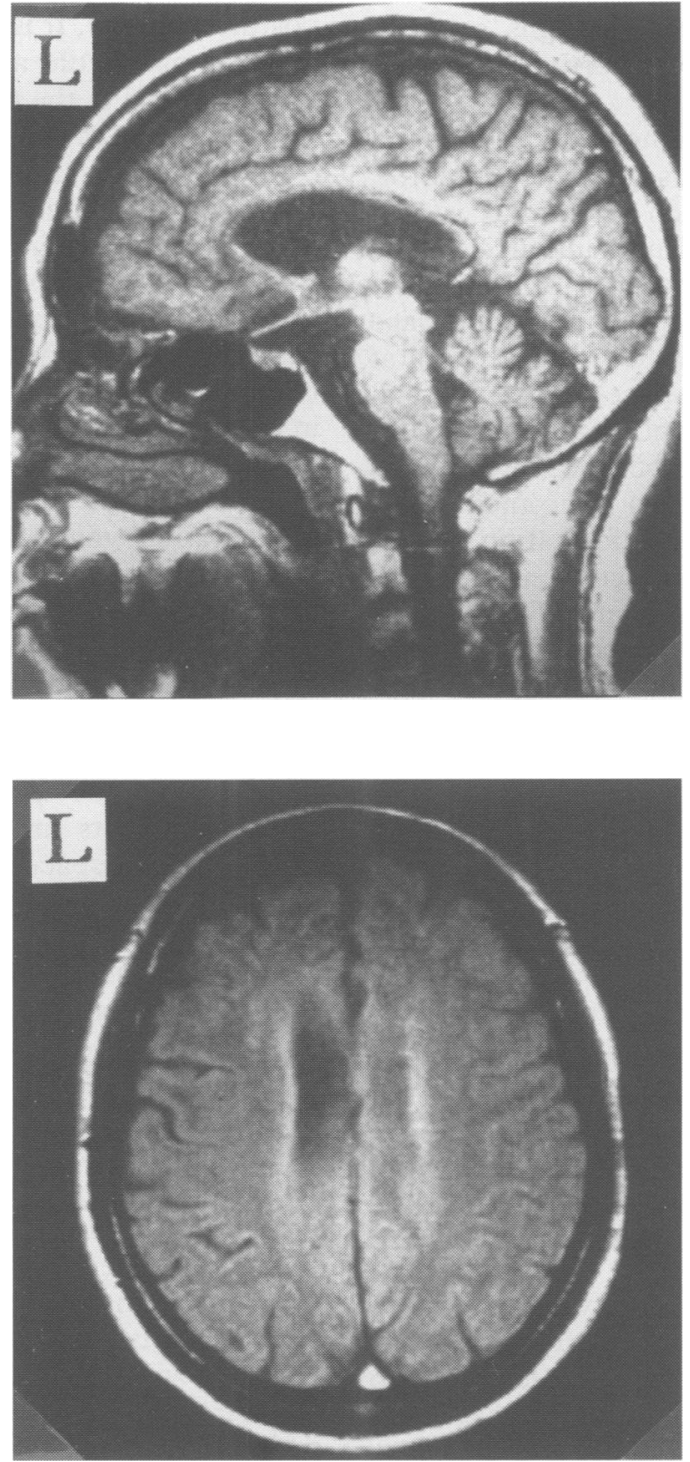

guiding the left hand to a correct position. Detailed examination on this aspect led us to the discovery of crossed avoiding reaction.

\section{Crossed avoiding reaction}

The crossed avoiding reaction was first noted 8 months after the onset. In November 1988 , one of us (TN) tested the patient's ability to grasp a stimulus held by the examiner in three dimensional space. With the right hand she showed no difficulty in either hemispace. With the left hand she was able to reach and grasp the stimulus placed in the left hemispace easily and without hesitation but could not reach it if it was placed in the right hemispace relative to her body axis including the median line. The left hand stiffened, the left shoulder jerked up, and the trunk stretched backward (fig 3). The harder she tried, the higher the muscle tension rose.

If, however, the right hand was mobilised to grasp the stimulus, the left hand was promptly relieved of its immobilisation, and moved in unison with the right hand to reach it; or, occasionally, the left hand would suddenly start moving by itself. This avoiding reaction did not occur in natural daily situa-

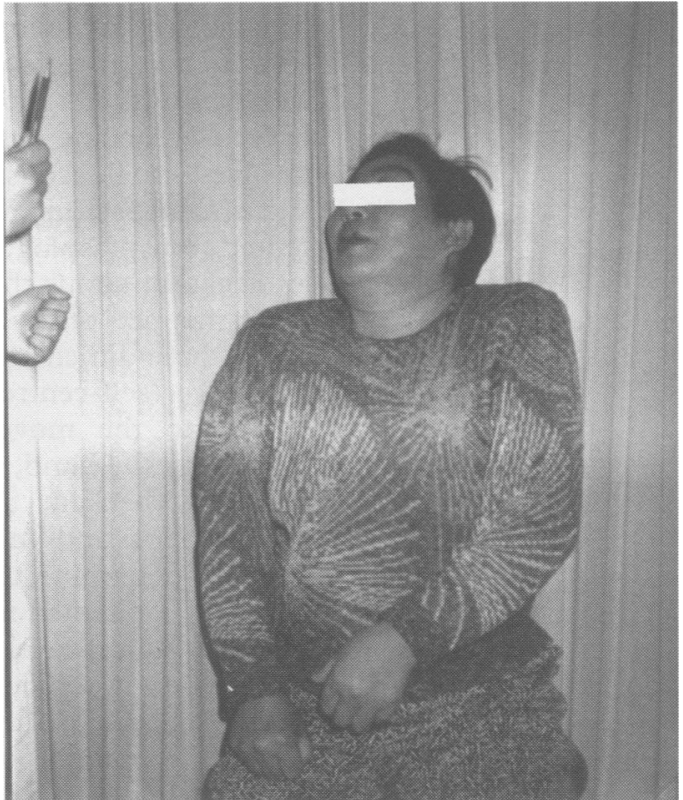

Figure 3 The patient's left arm demonstrates crossed avoiding reaction to a stimulus presented for grasping in the right hemispace.

tions. However, it was observed when she was drawing a picture with the left hand under instruction not to use the right hand. When she tried to delineate the right end of the picture in the right hemispace, the left hand stiffened unable to move. This symptom could be constantly elicited in the test situation for about ten months.

A follow up examination at three years revealed persistence of crossed avoiding reaction. Further, when she was requested to reach and grasp a ringing bell with the eyes closed, the left hand reacted in the same way as to the visual stimulus. Crossed avoiding reaction was also observed in the right hemispace with the left lower limb when she was asked to kick a ball.

\section{Discussion}

The patient's symptoms with respect to the goal reaching movement of the hands in the left and right hemispace are consistent with crossed avoiding reaction described by Lechevalier et al. ${ }^{1}$ It was impossible for her left hand to cross the median line of the body axis to reach a stimulus located in the right hemispace. In addition she showed three new features not previously described: (1) sudden facilitation of the left hand immobility either with or without assist from the right hand; (2) occurrence of the reaction without vision, and (3) occurrence only in the unnatural test situation.

Lechvalier and his colleagues argued that crossed avoiding reaction was a kind of disconnection syndrome resulting from a complex of lesions made up of damage to the corpus callosum and intrahemispheric injuries. ${ }^{1}$ This interpretation was also supported by Kashiwagi and others. ${ }^{2}$ Our patient had major lesions in the corpus callosum and the left cingulate gyrus. Taking account of 
concurrence of many clinical signs attributable to hemispheric disconnection, we agree with this interpretation.

Goal reaching movement of the hand involves visual, acoustic, somaesthetic, motor, and spatial factors. When a stimulus is presented for grasping, visual and/or acoustic information about the position of the stimulus is mapped on to a representational image of the three dimensional space with the self at its centre (self-centred space), which is then related to the movement of the hand. In mobilising the hand, the position of the stimulus is checked against the memorised somaesthetic and motor information of the hand to determine the direction and magnitude of the planned movement. Disruption of functions at any level of this complex network could result in difficulty in reaching. It is supposed that the quality of the difficulty is different depending on the nature of the impairment.

With regard to motor factors, her left hand showed no weakness, rigidity, dystonia, or ataxia at the time when crossed avoiding reaction was present. She had left sided apraxia but it is difficult to explain crossed avoiding reaction in terms of apraxia since it has never been observed to occur in unilateral space.

As for visual factors, left hemispatial neglect was present, but it was detected not in the left hand but in the right hand task. This is contrary to the left right relationship characterising crossed avoiding reaction. Crossed avoiding reaction is clearly different from optic ataxia ${ }^{3}$ or visuomotor ataxia, ${ }^{4}$ both of which are characterised by errors in reaching and not the impossibility of reaching. Also the present crossed avoiding reaction could also be elicited with an auditory stimulus.

Hand movement around the body is performed based on spatial information originating from two different sources-that is, extrapersonal spatial perception and personal spatial perception. ${ }^{5}$ Extrapersonal spatial perception deals with extracorporeal space where a target is perceived in relation to many other objects, while personal spatial perception deals with intracorporeal space where the hand movement is perceived in relation to other body parts. Information about the position of a target stimulus obtained through extrapersonal spatial perception is matched with information about the present status of the hand position obtained through the personal space perception system.

For goal reaching to succeed, another system which regulates motion in space and posture comes into play. This system is closely related to personal spatial perception and works for locating and mobilising the body, especially the hands. Sakata, ${ }^{6-8}$ in the series of studies with monkey brains, found a group of cells that synthetically processed sensory information from the skin and joints to provide tactile discernment of the spatial positions and movements of objects, as well as recognition of the posture pattern as a whole, and suggested such cells serve as the functional basis of senses for tactile space and posture patterns. It is proposed that this three dimensional tactile and postural information is transmitted to the premotor area, to play an important role in spatial posture regulation.

Postural regulation of the hands necessary for reaching is limited to the space outside of the body but within the reach of the hands. This "manual" space is biologically very important for humans to manipulate tools. Functional coordination between intracorporeal space and extracorporeal space is realised in this hypothetical space. We further hypothesise that our patient's bilateral manual space is represented in the left hemisphere but only unilateral left manual space is represented in the right hemisphere. If the link between the right and left hemisphere is disrupted by damage to the corpus callosum, the right hand's spatial operation would not be affected because its bilateral manual space is controlled by the left hemisphere. But the left hand would be prevented from the full expression of its function because its operation in the left space is controlled by the right hemisphere, while operation in right space is not permitted.

Why did the crossed avoiding reaction not occur in the natural daily situation? This so called automatico-voluntary dissociation ${ }^{9}$ has long been observed in aphasia and apraxia and regarded as a sign of higher level disturbance. Some of the callosal disconnection syndromes such as left hand apraxia also comply with this rule. ${ }^{10}$ It probably follows that the isolated left hand reaching task into the right hemispace in the test situation was a very artificial and demanding one which required special concentration. The fact that the right hand participation often resulted in facilitation of the left hand crossed avoiding reaction may mean that bilateral behaviour is a more natural and less demanding condition in which the left hand control from the right hemisphere becomes less necessary, or the left hand reaching is either facilitated ipsilaterally from the left hemisphere or controlled through pathways outside the damaged callosum.

Although the nature of this deficit is qualitatively different from motor dexterity and related more to spatial function, the pattern of right left asymmetry of the hand's performance suggests a close relation to handedness. The right hand motor dominance might have contributed to development of bilateral "manual space" in the left hemisphere to further facilitate its dexterity.

The substance of this paper was presented at the 14th annual meeting of the Neuropsychological Association of Japan at Nagoya in September 1990.

1 Lechevalier B, Andersson JC, Morin P. Hemispheric disconnection syndrome with a "crossed avoiding" reaction in a case of Marchiafava-Bignami disease. $₹$ Neuro Neurosurg Psychiatry 1977;40:483-97.

2 Kashiwagi T, Kashiwagi A, Eiko N, et al. A case of crossed avoiding reaction (abstract). Higher Brain Function Res. 1987;7:78.

3 Balint RL. Seelenlahmung des "Schauens", optische Ataxie, räumlichä Störung des Aufmerksamkeit. Monatsschr Psychiat Neurol 1909;25:51-81.

4 Rondot P, De Reconde J, Ribadeau Dumas JL. Visuomotor ataxia. Brain 1977;100:355-76. 
5 Semmes J, Weinstein S, Ghent L, Teuber H-L. Correlates of impaired orientation in personal and extrapersonal of impaired orientation in persc

6 Sakata H. Parietal association areas and space vision. $A d v$ Neurol Sci 1983;27:809-21. (in Japanese)

7 Sakata H. Parietal association areas and the voluntary movement. Adv Neurol Sci 1984;28:112-22. (in Japanese)
8 Sakata H. The activity of parietal lobe. Clin Neurosci 1985;4:22-9. (in Japanese)

9 Alajouanine T. Baillarger and Jackson; the principle of Baillarger-Jackson in aphasia. $\mathcal{F}$ Neurol Neurosurg

10 Yamadori A, Osumi Y, Imamura T, Mitani Y. Persistent left unilateral apraxia and a disconnection theory. Behav Neurol 1988;1:11-22. 